\title{
AOTD-Reisestipendium 2018: zu Besuch bei Jan Lindahl, Helsinki
}

\author{
Mohamed Omar
}

Mithilfe des Reisestipendiums der AOTrauma Deutschland wurde mir die einmalige Gelegenheit geboten, einerseits meine unfallchirurgischen Kenntnisse um neue Erfahrungen zu erweitern, andererseits aber auch Strukturen und Herangehensweisen der medizinischen Versorgung eines anderen Landes kennenzulernen.

Aufgrund meines persönlichen Interesses an der traumatologischen Beckenchirurgie entschied ich mich für den Besuch von Jan Lindahl im Universitätsklinikum in Helsinki, Finnland. Wir lernten uns im Rahmen des AO-Kurses „Acetabular and Pelvic Fracture Management“ in Graz kennen, bei dem er als einer der 3 Vorsitzenden fungierte. Schon während des Kurses hatte man auf unkomplizierte Weise die Möglichkeit, aus erster Hand von seinem unglaublichen Erfahrungsschatz in der Beckenchirurgie zu profitieren. Den Interessierten der Beckenchirurgie ist er als Mitverfassers des Werkes „Acetabular Fractures“ vom Thieme Verlag sicherlich ein Begriff. In seiner Laufbahn hat er insgesamt über 800 Acetabulumfrakturen versorgt.

Bereits die Organisation meines Besuchs verlief vollkommen unkompliziert und barg keinerlei bürokratischer Hürden. Meine Ankunft in Helsinki dagegen bedurfte aufgrund der witterungsbedingten Umstände einiger Gewöhnung: Temperaturen zwischen -10 und $-20^{\circ} \mathrm{C}$, begleitet von erheblichen Schneefällen, waren in dieser Zeit die Regel und die Witterung sollte sich dabei merklich auf das anstehende Versorgungsspektrum auswirken.

Das Universitätsklinikum in Helsinki war noch vor Kurzem an mehren Standorten vertreten, die nach und nach in einem Neubau zusammengeführt worden waren. Die Traumatologie/Orthopädie soll erst in etwa 3 Jahren in den Neubau integriert werden, sodass sich die Abteilung aktuell noch in den etwa 80 Jahre alten Räumlichkeiten im Stadtteil Töölö befindet, wohingegen ein 2., kleinerer Standort etwa 20 km entfernt in der Nähe des Flughafens im Stadteil Vantaa beheimatet ist. Hier werden in 2 Operationssälen in erster Linie elektive Eingriffe mit einem orthopädischen Schwerpunkt durchgeführt. Der Standort in Töölö beherbergt neben der Traumatologie/Orthopädie auch die Abteilungen der Neurochirurgie und Plastischen Chirurgie. Die Traumatologie/Orthopädie untergliedert sich wiederum in die Sektionen „Allgemeine Traumatologie“, „Obere Extremität“, „Becken und Untere
Extremität“, „Wirbelsäule“, „Endoprothetik“ und „Tumororthopädie“. Diese Aufteilung ermöglicht eine qualitativ hochwertige Versorgung, da Verletzungen mit höherer Komplexität expertenbezogen versorgt werden können. Neben den Notfällen führen die einzelnen Sektionen hierbei auch die elektiven Fälle ihrer entsprechenden Subspezialisierung durch. Als eine der wenigen Spezialkliniken im Land werden hier über die Notaufnahme nur Fälle aufgenommen, die entweder durch den Rettungsdienst vorgestellt oder aber im Vorfeld durch einen externen Arzt aufgrund der Fallschwere überwiesen werden.

Neben Jan Lindahl, als Leiter der Sektion „Beckenchirurgie und Untere Extremität“, sind weitere 7 Fachärzte und 3 Assistenten in dem Team beschäftigt. Jeweils 1 Fachund 1 Assistenzarzt führen hierbei im wöchentlichen Turnus eine 20-Betten-Station, auf der sich ausschließlich die eigens operierten Patienten befinden. In der Abteilung ist es üblich, dass lediglich Assistenten mit einem abgeschlossenem Common Trunk beschäftigt werden. Anders als der Common Trunk in Deutschland sieht dieser in Finnland eine 3-jährige Ausbildung in den großen chirurgischen Fächern vor, sodass entsprechend alle Assistenten bereits operative Expertise besitzen und Eingriffe gemäß ihres Ausbildungsstandes selbstständig durchführen können/dürfen, ohne dass jedoch eine Facharztpflicht bestünde. Das ungleiche Verhältnis von Fach- zu Assistenzärzten mit einem Überwiegen von Fachärzten mag für deutsche Verhältnisse sehr ungewöhnlich sein, begründet sich aber in der vollkommen anderen Herangehensweise. Sowohl auf persönlicher als auch fachlicher Ebene werden sehr flache Hierarchien gelebt. So führt selbst der Leiter der Abteilung z. B. Vordergrunddienste in der Notaufnahme oder im OP durch. Dies sei ein historisches Relikt der dortigen Unfallchirurgie und gehöre einfach zum guten Ton eines Abteilungsleiters.

In insgesamt 7 Operationssälen werden die ca. 25-30 traumatologischen und orthopädischen Eingriffe durchgeführt, wovon insgesamt 4 Säle theoretisch für 24 Stunden besetzt werden können. Die Atmosphäre im OP war durchweg sehr positiv und geprägt von guter interprofessioneller Zusammenarbeit. Interessanterweise werden für die Lagerung, Vor- und Nachbereitung der Patienten sowie die Bedienung der Bildwandler eigens ausgebildete Kräfte eingesetzt, sodass das ärztliche Personal erheblich entlastet wird. Historisch bedingt hat es sich allerdings 


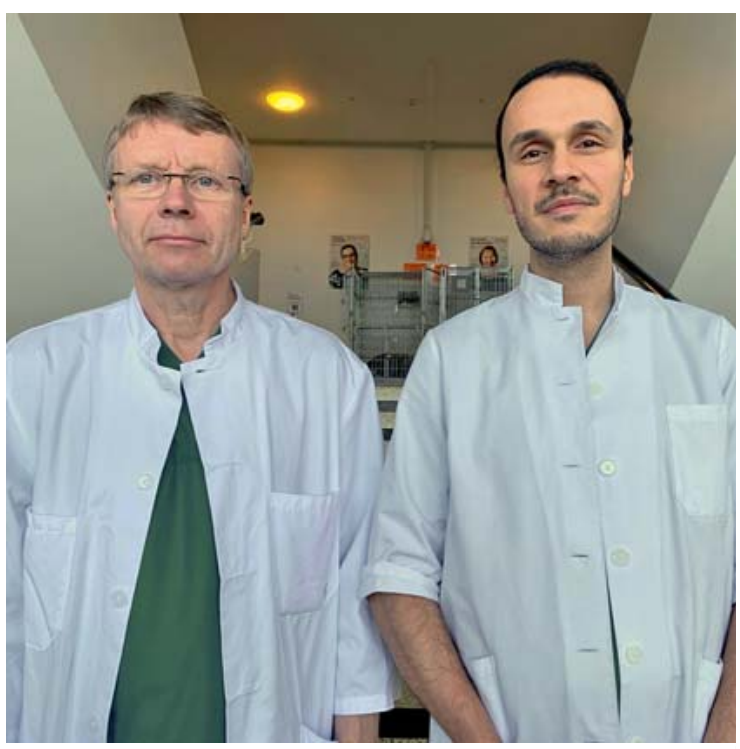

- Abb. 1 Jan Lindahl und Mohamed Omar. Quelle: Mohamed Omar.

eingebürgert, dass die Finnen die meisten Eingriffe ohne eine ärztliche Assistenz durchführen, sodass selbst komplexe Beckeneingriffe mitunter nur mit der instrumentierenden Schwester durchgeführt werden. Entsprechend positiv wurde mein Mitwirken im OP-Saal begrüßt.

Die eingangs erwähnten Witterungsverhältnisse bescherten der Klinik während meiner Anwesenheit eine überdurchschnittlich hohe Anzahl an Traumafällen. Typischerweise werden jährlich etwa 100-150 Beckenfrakturen operativ versorgt. In den 3 Wochen meines Aufenthalts waren es alleine 20 Eingriffe mit einem überwiegenden Anteil an Acetabulumfrakturen höherer Komplexität. Anders als in Deutschland sind die typischen Verletzungsursachen in Finnland Stürze aus großen Höhen und weniger Verkehrsunfälle. Der massive Schneefall hatte die Leute bewogen, ihre Dächer vom Schnee zu befreien, was in drei Viertel der Fälle den entsprechenden Traumamechanismus bedingte. Aufgrund der hohen traumatologischen Fallzahlen musste daher ein Großteil der elektiven Eingriffe in dieser Zeit abgesetzt werden.

Von der Versorgungsstrategie bis hin zur Durchführung band mich Herr Lindahl intensiv in die Fälle mit ein. Die operative Versorgung der Acetabulumfrakturen erfolgte bei anteriorem Zugehen ausschließlich über den modifizierten Stoppa-Zugang (intrapelvic approach) mit oder ohne iliakales Fenster, wohingegen von dorsal standardmäßig der Kocher-Langenbeck-Zugang gewählt wurde. Die anderen geläufigen Zugänge wie der ilioinguinale, iliofemorale oder der Pararectus-Zugang spielten wie die chirurgische Hüftluxation keine Rolle. In zahlreichen Jahren seines Schaffens hatte Jan Lindahl letztlich den Intrapelvic Approach als sein Arbeitspferd für die anteriore

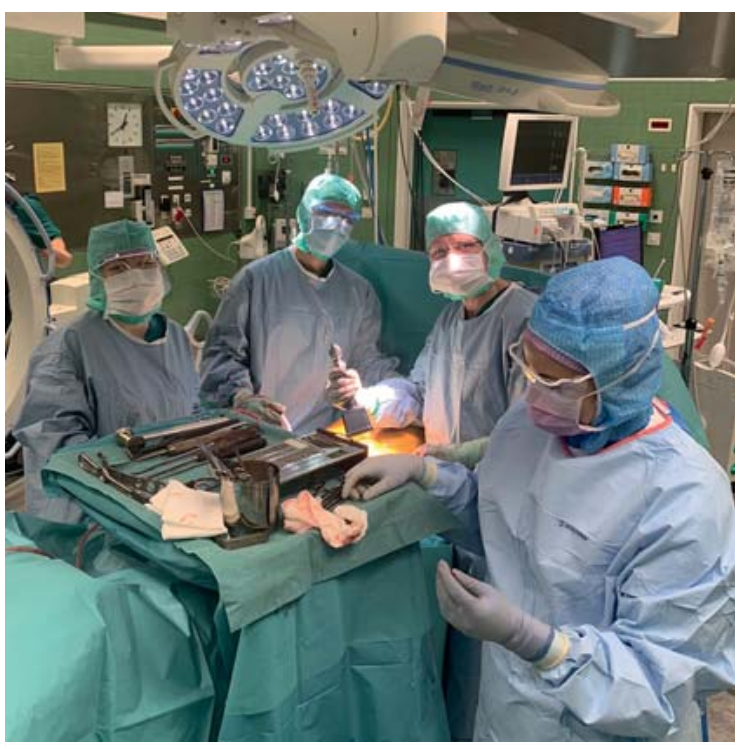

- Abb. 2 Im OP. Quelle: Mohamed Omar.

Versorgung erklärt, da er seiner Meinung nach mit geringstem Kollateralschaden bei gleichzeitig bester Visualisierbarkeit einhergeht. Entsprechend versiert und lehrreich liefen die Eingriffe ab. Besonders positiv erwähnenswert ist die Art und Weise von Herrn Lindahl, mir die einzelnen Schritte, Techniken und Pitfalls zu erklären, sodass ich viele Aspekte für meine Tätigkeit mit nach Hause nehmen konnte. Die vielen Beckenfrakturen waren für mich Fluch und Segen zugleich: Einerseits konnte ich in kürzester Zeit viele Eindrücke sammeln und verfestigen, andrerseits blieb kaum Zeit, die Versorgungsstrategien bei anderen Verletzungen kennenzulernen.

Neben den operativen Erfahrungen bot die Abteilung hervorragende Weiter- und Fortbildungsmöglichkeiten sowie regelmäßige Diskussionsrunden. Zum einen ist die sehr umfangreiche und lehrreiche Frühbesprechung zu erwähnen, die nicht nur als Fallvorstellung, sondern auch als Weiterbildung für die Assistenten verstanden und gelebt wurde. Darüber hinaus wurden zum anderen einmal wöchentlich komplexe Fälle im Plenum besprochen, um einen gemeinsamen Konsens für deren Versorgung zu finden. Um am wissenschaftlichen Puls der Zeit zu bleiben, fand 2-mal wöchentlich ein Journal-Club statt, in dem hochrangige internationale Arbeiten vorgestellt, kritisch diskutiert und ihre Umsetzbarkeit in den eigenen Reihen erörtert wurden.

Nicht nur fachlich, sondern auch menschlich war der Aufenthalt eine außergewöhnliche Erfahrung. Ich wurde vom ersten Tag an hervorragend integriert. Bemerkenswert war, dass trotz zahlreicher Verpflichtungen jedes Einzelnen 2-mal gemeinsame Dinner mit dem gesamten 
Team arrangiert werden konnten und dann auch der persönliche Austausch in den Vordergrund trat.

Mein großer Dank gilt der AOTrauma Deutschland für die Ermöglichung dieses Aufenthalts sowie Jan Lindahl, der mir proaktiv außerordentliche und bleibende Eindrücke der Beckenchirurgie vermittelte. Die gesammelten Erfahrungen werden mich fachlich weiterbringen und der Einblick in die finnische Herangehensweise sowie die dortigen medizinischen Strukturen meine zukünftigen konzeptuellen Überlegungen positiv beeinflussen.
Korrespondenzadresse

PD Dr. Mohamed Omar

omar.mohamed@mh-hannover.de

Bibliografie

DOI https://doi.org/10.1055/s-0037-1599606

OP-JOURNAL 2019; 35: 206-208 @ Georg Thieme Verlag KG Stuttgart · New York ISSN 0178-1715 